University of Nebraska - Lincoln

DigitalCommons@University of Nebraska - Lincoln

10-24-2007

\title{
A high repetition rate time-of-flight electron energy analyzer
}

\author{
Shawn A. Hilbert \\ University of Nebraska-Lincoln, shilbert@berry.edu \\ Brett E. Barwick \\ University of Nebraska-Lincoln, brett.barwick@trincoll.edu \\ M. Fabrikant \\ University of Nebraska - Lincoln \\ Cornelis J. Uiterwaal \\ University of Nebraska - Lincoln, cuiterwaal2@unl.edu \\ Herman Batelaan \\ University of Nebraska - Lincoln, hbatelaan@unl.edu
}

Follow this and additional works at: https://digitalcommons.unl.edu/physicsuiterwaal

Part of the Physics Commons

Hilbert, Shawn A.; Barwick, Brett E.; Fabrikant, M.; Uiterwaal, Cornelis J.; and Batelaan, Herman, "A high repetition rate time-of-flight electron energy analyzer" (2007). C.J.G.J. Uiterwaal Publications. 3. https://digitalcommons.unl.edu/physicsuiterwaal/3

This Article is brought to you for free and open access by the Research Papers in Physics and Astronomy at DigitalCommons@University of Nebraska - Lincoln. It has been accepted for inclusion in C.J.G.J. Uiterwaal Publications by an authorized administrator of DigitalCommons@University of Nebraska - Lincoln. 


\title{
A high repetition rate time-of-flight electron energy analyzer
}

\author{
S. A. Hilbert, ${ }^{\text {a) }}$ B. Barwick, M. Fabrikant, C. J. G. J. Uiterwaal, and H. Batelaan \\ University of Nebraska-Lincoln, Lincoln, Nebraska 68588-0111, USA
}

(Received 20 August 2007; accepted 2 October 2007; published online 24 October 2007)

\begin{abstract}
We demonstrate a time-of-flight electron energy analyzer that operates at an $80 \mathrm{MHz}$ repetition rate. The analyzer yields an energy resolution of $40 \mathrm{meV}$ for $3 \mathrm{eV}$ electrons. The energy resolution limit is dominated by the detector time (or temporal) resolution. With a currently available detector with a temporal resolution of $100 \mathrm{ps}$, we predict an energy resolution of less than $1 \mathrm{meV}$ for $200 \mathrm{meV}$ electrons. This makes high repetition rate time-of-flight energy analyzers a promising low-technology alternative to current state-of-the-art techniques. (C) 2007 American Institute of Physics. [DOI: 10.1063/1.2801523]
\end{abstract}

Many investigations of low-energy electron processes require high energy resolution. ${ }^{1}$ For instance, vibrational Feshbach resonances in biological molecules have very long lifetimes, yielding narrow electron resonances. ${ }^{2}$ Determining the width of these resonances will give the lifetimes of these low-energy channels and could shed light on possible causes for DNA strand breaks. ${ }^{3}$ Electron resonances also occur in metallic clusters. ${ }^{4}$ Determining properties of such resonances could lead to information about the evolution from atomic and molecular effects to bulk properties of solids. ${ }^{5}$ There are difficulties in finding low-energy resonances. For instance, ozone was shown to have a zero-energy resonance that may have been a decay channel to ozone depletion in the ionosphere. ${ }^{6}$ This result was shown to be erroneous ${ }^{7}$ and displays the difficulty in identifying low-energy resonances. High repetition rate time-of-flight energy analyzers with high energy resolution can give insight into, and provide a different method for, determining properties of these resonances.

Typically, energy analyzers, such as hemispherical ${ }^{8}$ and retarding field analyzers, ${ }^{9}$ are preferred over time-of-flight (TOF) methods at low energies, because they have better energy resolutions. Such methods have provided $0.2 \mathrm{meV}$ resolution for $0-200 \mathrm{meV}$ electrons. ${ }^{10}$ Such resolutions are reached with measuring times of about a day. ${ }^{11}$ Data acquisition times for TOF systems are potentially shorter than those of scanning energy analyzers, because all energies are acquired simultaneously. The trade-off is that current TOF methods are limited in energy resolution by slow emission processes and low repetition rates of the source. Slow emission processes cause the flight times to be more affected by time of emission rather than electron energy. Low repetition rates require many electrons to be emitted for each pulse to maintain acceptable count rates. ${ }^{12}$ The Coulomb interactions between electrons emitted from the same pulse hinder the determination of the electron energy from its time of flight. ${ }^{13}$ The use of a femtosecond electron source with a high repetition rate produces reasonable count rates without the difficulties usually associated with TOF techniques.

Laser-induced femtosecond emission from a field emission tip takes less than $100 \mathrm{fs} .{ }^{14}$ Since the best temporal resolution currently available for commercial electron detectors is better than $100 \mathrm{ps},{ }^{15}$ the laser-induced femtosecond emission does not limit the energy resolution. The laser's $80 \mathrm{MHz}$

\footnotetext{
${ }^{\text {a) }}$ Electronic mail: shilber1@ bigred.unl.edu
}

repetition rate enables high count rates with less than one electron per pulse, preventing Coulomb broadening.

The energy analyzer is comprised of a retarding field analyzer plus a drift tube region. Figure 1 shows the front and back views as well as a schematic of the drift tube. A voltage on the half-inch $(12.7 \mathrm{~mm})$ diameter drift tube controls the electron's kinetic energy inside the tube. The drift tube is held inside a vacuum flange by an insulated aluminum mount and is magnetically shielded by two wrapped layers of $\mu$-metal with $0.2 \mathrm{~mm}$ thickness. This shielding reduces the background magnetic field to $38 \mathrm{mG}$ inside the drift tube. The front of the tube has a fine copper mesh ( $25 \mu \mathrm{m}$ diameter; $490 \mu \mathrm{m}$ spacing) while the back has a thicker mesh (240 $\mu \mathrm{m}$ diameter; $690 \mu \mathrm{m}$ spacing.) A $3.0 \mathrm{~cm}$ diameter aluminum cap with a $3 \mathrm{~mm}$ diameter pinhole covers the front mesh. The cap is insulated from the tube and grounded. The tube is positioned so the cap is $1.5 \mathrm{~cm}$ from the tip.

The electrons are emitted by focusing laser pulses on a field emission tip ${ }^{14}$ with a voltage of $V_{0}=V_{\text {tip }}=-75 \mathrm{~V}$. The

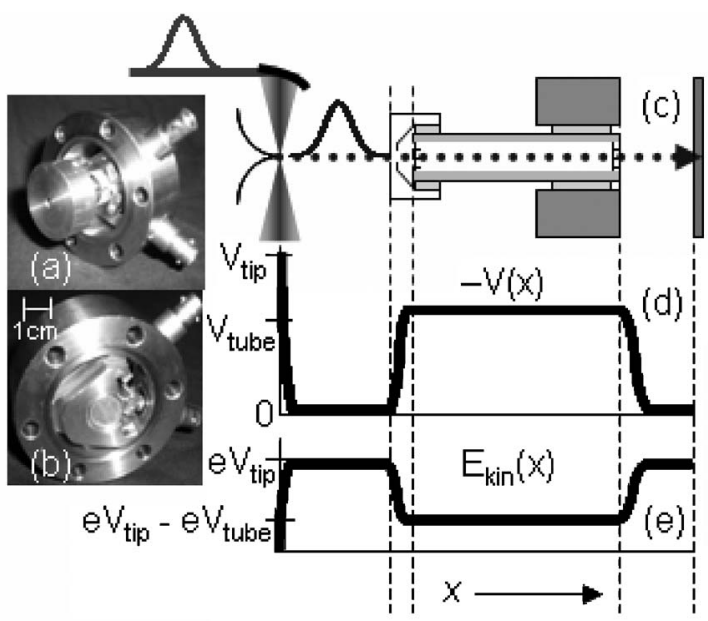

FIG. 1. Drift tube. (a) The front of the drift tube is covered by a large aluminum cap which defines the ground. Directly behind the cap is a fine mesh (not visible), which defines the potential of the tube for the incoming electrons. (b) The back of the tube has a thicker mesh which also defines the tube potential for the electrons. The tube mount protrudes slightly past the end of the tube, causing a sharp potential gradient at the end of the tube. (c) The electrons are emitted from the tip, enter through the cap on the drift tube, and travel through the tube. After exiting the tube, the electrons hit the detector plate and are recorded by the data acquisition system. (d) A graph of the electrostatic potential along the electron trajectory. For convenience, we show $-V(x)$. (e) The electron kinetic energy as a function of position. 


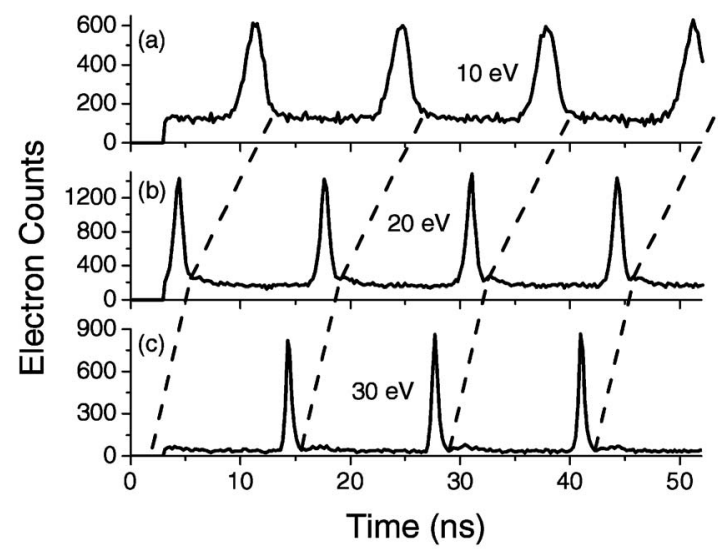

FIG. 2. Experimental time-of-flight spectra for three different electron energies: (a) $10 \mathrm{eV}$, (b) $20 \mathrm{eV}$, and (c) $30 \mathrm{eV}$. Each spectrum has multiple peaks, because not every laser pulse frees an electron. For decreasing energies, the peaks shift to later times, while their widths broaden. Dashed lines are an eye's guide to peak shift.

laser trigger starts the time-to-amplitude converter (TAC) we use to record flight times. Once emitted, the electrons experience a radial electric field. The transit time for a distance $x$ is

$$
t=x\left(\frac{2 e V_{0}}{m}\right)^{-1 / 2}\left[1+\frac{r_{\text {tip }}}{2 x} \ln \left(\frac{x}{r_{\text {tip }}}\right)\right],
$$

where $m$ is the electron mass and $r_{\text {tip }}$ is the tip radius. Since the radius of the tip is much smaller than the electron's travel distance, the transit time may be approximated by

$$
t=x\left(\frac{2 e V_{0}}{m}\right)^{-1 / 2} \text {. }
$$

The transit time from the tip to the cap is found by using $x=1.5 \mathrm{~cm}$.

After going through the cap's pinhole, the electrons decelerate and reach the front mesh of the drift tube. This deceleration region is very short compared to the tube length and the time spent in this region is ignored. The front mesh has the same potential as the tube, $V_{\text {tube }}$. During the deceleration, the electrons lose kinetic energy equal to $e\left|V_{\text {tube }}\right|$. The electrons travel through the $4.6 \mathrm{~cm}$ long tube with a kinetic energy of $e V_{0}=e\left|V_{\text {tip }}-V_{\text {tube }}\right|$.

Upon reaching the end of the tube, the electrons are accelerated to the original energies that they had before they entered the tube by the electric field between the back mesh and the grounded detector plate $2.7 \mathrm{~cm}$ from the mesh. In this region, electrons are quickly accelerated because the grounded tube mount extrudes slightly past the end of the tube. The acceleration region is small compared to the travel distance, so Eq. (2) is a good approximation for the travel time in this region. Electron detection provides the stop to the TAC. The time between TAC start and stop gives the time of flight.

The uncertainty in the time of flight is related to the uncertainty in the travel distance, $\Delta x$, and the tip voltage, $\Delta V$

$$
\Delta t=\sqrt{\frac{m}{2 E}\left[(\Delta x)^{2}+\frac{x^{2}(e \Delta V)^{2}}{4(e V)^{2}}\right]} .
$$

There are two types of uncertainties in our system: absolute and relative. The absolute uncertainties, travel length and
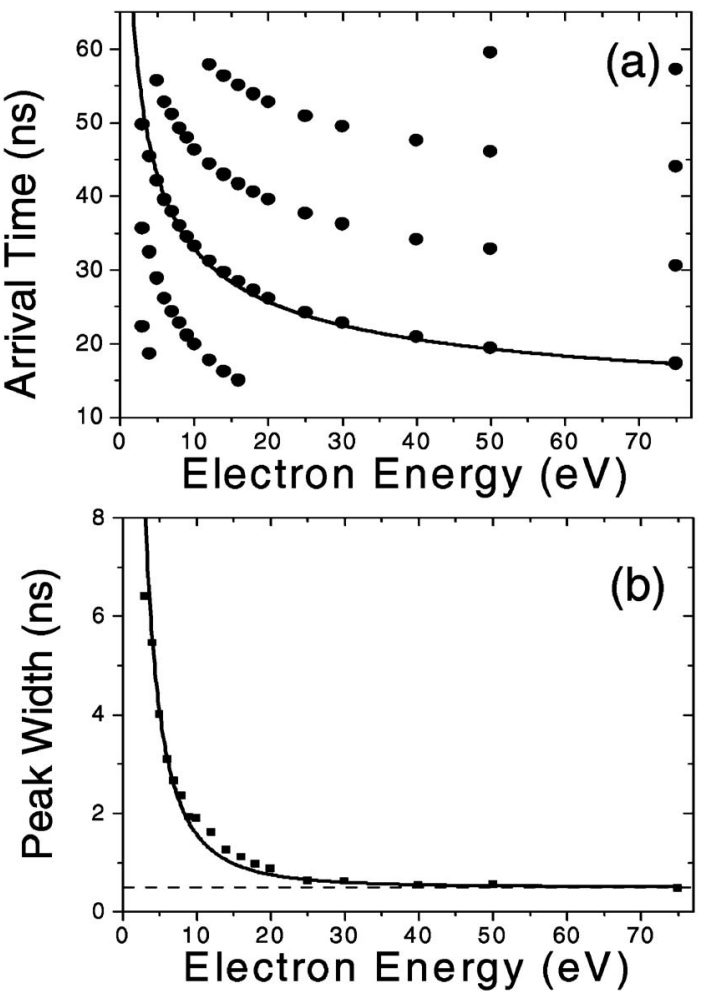

FIG. 3. Time-of-flight and pulse width. (a) The experimental time of flight (dots) is compared to the expected time of flight given from the simulation (solid curve). The experimental data have a free parameter in that the times actually measured are relative times, so the absolute scale can be shifted. Multiple flight times per energy appear because multiple peaks are seen per spectrum. (b) The experimental temporal peak width (dots) is compared to the peak width predicted by the simulation (solid curve). The temporal resolution of about $0.3 \mathrm{~ns}$ is given by the width observed for high energy electrons (dashed line).

voltage offset, are the same for each electron within a spectrum; their effect on the temporal resolution is minimal. Of the relative uncertainties, tip vibrations, tip voltage instability, and detector resolution, the latter dominates. The tip vibration (less than $10 \mu \mathrm{m}$ ) and the tip voltage ripple (about $5 \mathrm{meV}$ ) lead to a relative temporal uncertainty of less than $40 \mathrm{ps}$. The detector resolution is $\Delta t_{d}=300 \mathrm{ps}$ (see below) and gives an approximate energy resolution, $\Delta E$, of

$$
\Delta E=\frac{2 \Delta t_{d}}{t} E
$$

The time-of-flight spectra in Fig. 2 show that the temporal peak widths increase and the peak positions shift toward later times as the electron energy is decreased. Multiple peaks are found per spectrum because not every laser pulse produces an electron. The zero of the time axes of the individual spectra is not calibrated. The absolute zero is found by fitting multiple experimental flight times [Fig. 3(a)] to a simulation. The flight times [Fig. 3(a)] are determined by the peak positions found in the spectra (Fig. 2) for each energy. The peak widths [Fig. 3(b)] are defined from the spectra of Fig. 2 by taking the full width at half maximum. Finding peak widths becomes problematic for energies lower than $3 \mathrm{eV}$, when the peak width approaches the time between laser pulses, causing the electron pulses to merge. A lower laser repetition rate or a pulse picker could solve this issue at the expense of the electron count rate. 
The experimental data are compared to a simulation of the electron trajectories based on repeated application of Eq. (2) as previously described. Using a $0.7 \mathrm{eV}$ width Gaussian as the initial energy distribution fits the results well. The temporal resolution of the detection system is determined to be $0.3 \mathrm{~ns}$ from the peak widths found for high energies [dotted line in Fig. 3(b)] This detector resolution is convoluted with the simulated spectra to obtain the curve in Fig. 3(b), which agrees well with the experimental temporal peak width.

From Eq. (4), the system's energy resolution can be calculated. An energy resolution of about $\Delta E=40 \mathrm{meV}$ is found for $E=3 \mathrm{eV}$ electrons, for which the measured and calculated time of flight and time resolution are $t=49 \mathrm{~ns}$ and $\Delta t$ $=0.3 \mathrm{~ns}$, respectively. This resolution could be easily improved by using a longer drift tube ( $t$ would increase), though this would require more stringent magnetic shielding to prevent deflection and a lowered repetition rate to prevent electron pulse overlap.

Our results demonstrate that an $80 \mathrm{MHz}$ femtosecond electron source can be paired with TOF analyzers. With commercially available detectors with a temporal resolution better than $100 \mathrm{ps}$, ${ }^{15}$ magnetic shielding that is proven to work with $200 \mathrm{meV}$ electrons, ${ }^{10}$ and a drift tube of $5 \mathrm{~cm}$, Eq. (4) predicts an energy resolution of $0.2 \mathrm{meV}$. This resolution can be achieved while preventing Coulomb broadening by using a $2 \mathrm{MHz}$ repetition rate yielding one electron emitted per pulse (we observed emission rates of ten electrons per pulse.) With this repetition rate, $4 \mathrm{~h}$ of data acquisition leads to an estimated signal to noise ratio of 10 . These estimations dis- play promise for high repetition rate TOF analyzers to be competitive with currently used energy analyzers.

This material was based upon work supported by the National Science Foundation under Grants Nos. PHY0355235 and PHY-0653182. The authors would like to thank Paul Burrow for many fruitful discussions.

${ }^{1}$ H. Hotop, M.-W. Ruf, M. Allan, and I. I. Fabrikant, Adv. At. Mol. Opt. Phys. 49, 85 (2003).

${ }^{2}$ P. D. Burrow, G. A. Gallup, A. M. Scheer, S. Denifl, S. Ptasinska, T. Märk, and P. Scheier, J. Chem. Phys. 124, 124310 (2006).

${ }^{3}$ F. Martin, P. D. Burrow, Z. Cai, P. Cloutier, D. Hunting, and L. Sanche, Phys. Rev. Lett. 93, 068101 (2004).

${ }^{4}$ J.-P. Connerade, L. G. Gerchikov, and A. N. Ipatov, J. Phys. B 33, 5109 (2000).

${ }^{5}$ M. Bernath, O. Dragún, M. R. Spinella, H. Massmann, and J. M. Pacheco, Phys. Rev. A 52, 2173 (1995).

${ }^{6}$ G. Senn, J. D. Skalny, A. Stamatovic, N. J. Mason, P. Scheier, and T. D. Märk, Phys. Rev. Lett. 82, 5028 (1999).

${ }^{7}$ P. Cicman, J. D. Skalny, J. Fedor, N. J. Mason, P. Scheier, E. Illenberger, and T. D. Märk, Int. J. Mass. Spectrom. 260, 85 (2007).

${ }^{8}$ M. Allan, Phys. Scr., T 110, 161 (2004).

${ }^{9}$ P. E. Batson, Rev. Sci. Instrum. 57, 43 (1986).

${ }^{10}$ D. Klar, M. W. Ruf, and H. Hotop, Chem. Phys. Lett. 189, 448 (1992).

${ }^{11}$ M. Allan, Personal Communication (July 3, 2007).

${ }^{12}$ R. Ganter, R. J. Bakker, C. Gough, M. Paraliev, M. Pedrozzi, F. Le Pimpec, L. Rivkin, and A. Wrulich, Nucl. Instrum. Meth. Phys. Res. A $\mathbf{5 6 5}, 423$ (2006)

${ }^{13}$ B. J. Siwick, J. R. Dwyer, R. E. Jordan, and R. J. D. Miller, J. Appl. Phys. 92, 1643 (2002).

${ }^{14}$ B. Barwick, C. Corder, J. Strohaber, N. Chandler-Smith, C. Uiterwaal, and H. Batelaan, New J. Phys. 9, 142 (2007).

${ }^{15}$ H. Kiesel, A. Renz, and F. Hasselbach, Nature (London) 418, 392 (2002). 